\title{
Correlation between the Level of Urinary D-Glucaric Acid and the Degree of Activation of Masked Compound (FT-207) in Cancer Patients
}

\author{
Shigeru Matsuoka, Sakae Maezawa, Masayoshi Sakuma, \\ Sadao Ohira, Akira Wakui and Tatuo SaIto* \\ Department of Clinical Cancer Chemotherapy, The Research \\ Institute for Tuberculosis and Cancer, Tohoku University, \\ Sendai 980 and *Department of Internal Medicine, Cancer \\ Institute Hospital, Tokyo ${ }^{17} 0$
}

\begin{abstract}
Matsuoka, S., Maezawa, S., Sakuma, M., Ohira, S., Wakut, A. and Saito, T. Correlation between the Level of Urinary L-Glucaric Acid and the Degree of Activation of Masked Compound (FT-20\%) in Cancer Patients. Tohoku J. exp. Med., 1981, 135 (3), 281-290 — Phenobarbital stimulates the induction of liver microsomal drug-metabolizing enzyme, namely, cytochrome P-450, which enhances the rate of conversion of FT-207 to 5-FU, the active substance. When FT-207 is administered in combination with phenobarbital to cancer patients, the fluctuation in level of the drug-metabolizing enzyme, cytochrome P-450, should be taken into consideration. Therefore, it was investigated whether the urinary level of D-glucaric acid could be of value as an indicator for the evaluation of the activation of masked compounds, such as FT-207. The level of D-glucaric acid in urine was lower in cancer patients than in normal controls. The correlation between the level of urinary D-glucaric acid and that of 5-FU, which is an active metabolite of FT-207, in blood was statistically significant. The level of D-glucaric acid in urine was of use as an indicator for the evaluation of the activation of masked compounds, such as FT-207, in cancer chemotherapy. urinary D-glucaric acid excretion; 1-(2-tetrahydrofuryl)-5-fluorouracil (FT-207); 5-fluorouracil (5-FU); liver microsomal drug-metabolizing enzyme (cytochrome P-450); cancer chemotherapy
\end{abstract}

It has already been reported that FT-207, one of the masked compounds, is metabolized by liver microsomal drug-metabolizing enzymes (cytochrome P-450) to 5-FU and then shows an anticancer activity. Thus, it has been emphasized that, in the administration of FT-207, pretreatment with an inducer of cytochrome P450 , such as phenobarbital, is useful because the level of this drug-metabolizing enzyme is low in tumor-bearing hosts (Ohira et al. 1976a, 1978; Ohira 1977).

In clinical practice, however, it is difficult to measure the activity of cytochrome P-450 in cancer patients. Hunter et al. (1973) reported that a highly significant correlation was found between the total liver content of microsomal

Received for publication, July 17, 1980.

This paper is presented in commemoration of the retirement of Professor Tatuo Saito, the Director of the Research Institute for Tuberculosis and Cancer, Tohoku University, April 1978. 
enzyme cytochrome P-450 and the daily urinary excretion of D-glucaric acid in guinea pigs which possessed the glucuronic acid pathway as man does (Fig. 1).

In this paper, a study was made to find out whether the measurement of the level of D-glucaric acid in urine could be of value as an indicator for the evaluation. of the activation of masked compound in humans.

\section{Materials and Methods}

Subjects

Controls consisted of healthy 18 men and 13 women ranging in age from 26 to 66 years. Cancer patients who received no cancer chemotherapy comprised 29 men and women ranging in age from 22 to 83 years; namely 13 cases of stomach cancer, 6 cases of esophageal cancer, 2 cases of pancreatic cancer and 8 cases of cancer of miscelaneous origins, for example, lung cancer, colon cancer, cholangioma, breast cancer, malignant melanoma, lymphosarcoma and others.

A 24-hr urine specimen was collected and the urine was stored at $0^{\circ} \mathrm{C}$ when examined within $24 \mathrm{hr}$ after collection, or otherwise at $-20^{\circ} \mathrm{C}$.

Method of measurement of urinary D-glucaric acid

The level of urinary D-glucaric acid was determined by the method of Marsh (1963), namely by $\beta$-glucuronidase assay. The results were expressed in terms of $\mathrm{mg}$ glucaro-1,4lactone per $24 \mathrm{hr}$. Quantitation is based on the fact that glucaro-1, 4-lactone, which is produced when $\mathrm{D}$-glucaric acid is boiled at acid $\mathrm{pH}$, is a specific inhibitor of $\beta$-glucuronidase.

Reagents. (1) Standard substrate. $14 \mathrm{mg}$ of phenolphthalein mono- $\beta$-glucuronic acid sodium salt (mol. wt. 516.4, Sigma Chemical Co., St. Louis, Mo. 63178) was dissolved in 10 $\mathrm{ml}$ of acetate buffer (conc. $0.63 \mathrm{mM}$ ). (2) $0.125 \mathrm{M}$-acetic acid- $\mathrm{NaOH}$ buffer $(\mathrm{pH} 5.2)$. It was kept in the cold. (3) $\beta$-Glucuronidase (E.C. No. 3.2.1.311, from bovine liver, Type B-1, Sigma Chemical Co.). $10 \mathrm{mg}$ was dissolved in $10 \mathrm{ml}$ of acetate buffer. (4) 1,4-DGlucarolactone monohydrate (mol. wt. 210, Calbiochem, San Diego, Calif. 92112). (5) $3 \mathrm{~N}$ HCl. (6) $1 \mathrm{~N} \mathrm{NaOH.} \mathrm{(7)} 0.1 \mathrm{~N} \mathrm{NaOH}$.

Treatment of urine. A 24-hr urine was heated at $100^{\circ} \mathrm{C}$ for $40 \mathrm{~min} . \mathrm{pH}$ of the urine was adjusted with $3 \mathrm{~N} \mathrm{HCl}$ to 2.1 and then readjusted with $1 \mathrm{~N} \mathrm{NaOH}$ to 4.25 .

Incubation mixture. An incubation mixture $(4 \mathrm{ml})$ for urine samples included $1.0 \mathrm{ml}$ of a urine sample treated as above, $1.0 \mathrm{ml}$ of the substrate solution, $0.5 \mathrm{ml}$ of $\beta$-glucuronidase and $1.5 \mathrm{ml}$ of $0.125 \mathrm{M}$ acetate buffer (pH 5.2). An incubation mixture $(4 \mathrm{ml})$ for standard curve included $1.0 \mathrm{ml}$ of a solution of glucaro-1,4-lactone of various concentrations, $1.0 \mathrm{ml}$ of substrate solution, $0.5 \mathrm{ml}$ of $\beta$-glucuronidase and $1.5 \mathrm{ml}$ of $0.125 \mathrm{M}$ acetate buffer.

Measurement. A reference curve was prepared at each determination. The reaction mixture $(4 \mathrm{ml})$ was incubated for $60 \mathrm{~min}$ at $37^{\circ} \mathrm{C}$. Then $10 \mathrm{ml}$ of $0.1 \mathrm{~N} \mathrm{NaOH}$ was added to the mixture. After the sample was kept standing for $20 \mathrm{~min}$, absorbance at $550 \mathrm{~nm}$ was read with a Hitachi recording spectrophotometer, Model 124, and the quantity of the excreted urinary glucaro-1,4-lactone was determined with reference to a standard curve.

Estimation of serum FT-207 and 5-FU levels in cancer patients

Venous blood was taken from a total of 30 cancer patients $1,4,8$ and $12 \mathrm{hr}$ after intravenous administration of $800 \mathrm{mg}$ of FT-207. The blood level of FT-207 and that of its active metabolite (5-FU) were determined by bioassay (Staphylococcus aureus 209P) using the cup method (Fujita et al. 1972). The results were expressed in terms of $\mu \mathrm{g} / \mathrm{g}$. At the same time the level of urinary D-glucaric acid was determined and the effect of P-450 on the degree of FT-207 activation was evaluated indirectly. 


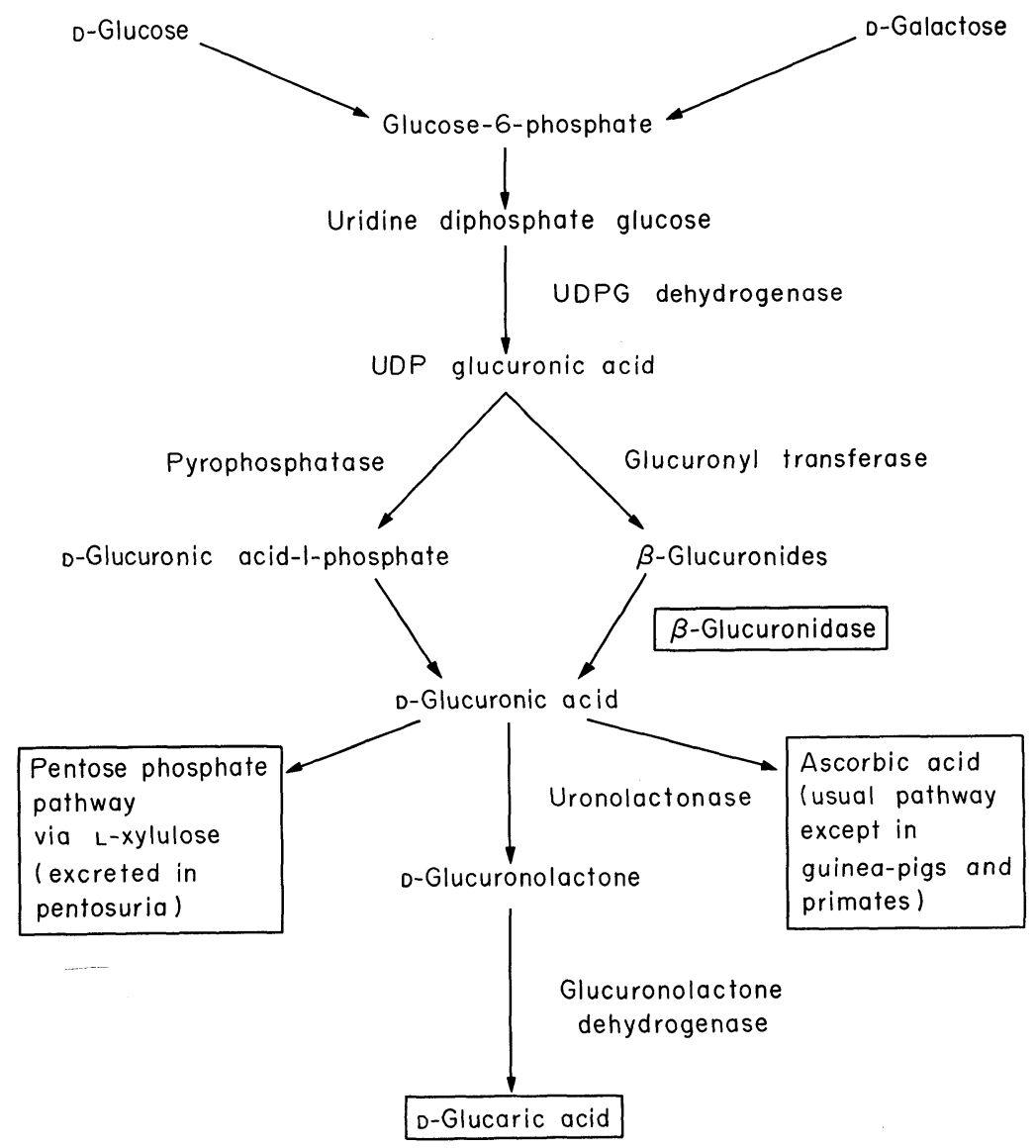

Fig. 1. Urinary D-glucaric acid excretion pathway (Hunter et al. 1973).

\section{Results}

The level of D-glucaric acid in urine of cancer patients

The results are summarized in Fig. 2. The mean value of urinary Dglucaric acid level in controls was $4.25 \mathrm{mg}$ glucaro-1,4-lactone per day (s.D. 1.34) in 18 men and 3.89 (s.D. 1.58) in 13 women. On the other hand, the mean value in cancer patients was as follows: 1.15 (s.D. 0.93) in 13 stomach cancer patients, 1.27 (s.D. 0.67) in 6 esophageal cancer patients, 0 (s.D. 0) in 2 pancreatic cancer patients and 1.89 (S.D. 1.22) in 8 other cancer patients.

Thus, it was found that the mean value of urinary D-glucaric acid level in cancer patients was lower than that in normal controls.

The correlation between urinary D-glucaric acid level and the degree of activation of FT-20\% in cancer patients

In Figs. 3, 4 and 5, the open circles (०) show cases which received no pretreatment, the closed circles $(\bullet)$ cases pretreated with phenobarbital $(200 \mathrm{mg} /$ 
day) for 3 consecutive days, and the triangles $(\boldsymbol{\wedge})$ cases pretreated with glutathione $(2.4 \mathrm{~g} /$ day) or vitamine $\mathrm{E}(1.5 \mathrm{~g} /$ day), and inhibitor of lipid peroxidation (Ohira et al. 1978).

In cancer patients who received FT-207, the correlation between urinary Dglucaric acid level and 5-FU level in blood $1 \mathrm{hr}$ after administration of FT-207 was statistically significant ( $n=30, r=0.701, p<0.001$, left in Fig. 3). The correlation after $4 \mathrm{hr}$ was also statistically significant $(n=30, r=0.726, p<0.001$, right in Fig. 3 ). However, the correlation after $8 \mathrm{hr}$ was not statistically significant $(n=30$, $r=0.333, p>0.1$, left in Fig. 4 ), and that after $12 \mathrm{hr}$ was statistically significant $(n=30, r=0.464, p<0.01$, right in Fig. 4). The correlation between the urinary D-glucaric acid level and the product of serum concentration and time $(\mu \mathrm{g} / \mathrm{g} \times \mathrm{hr})$

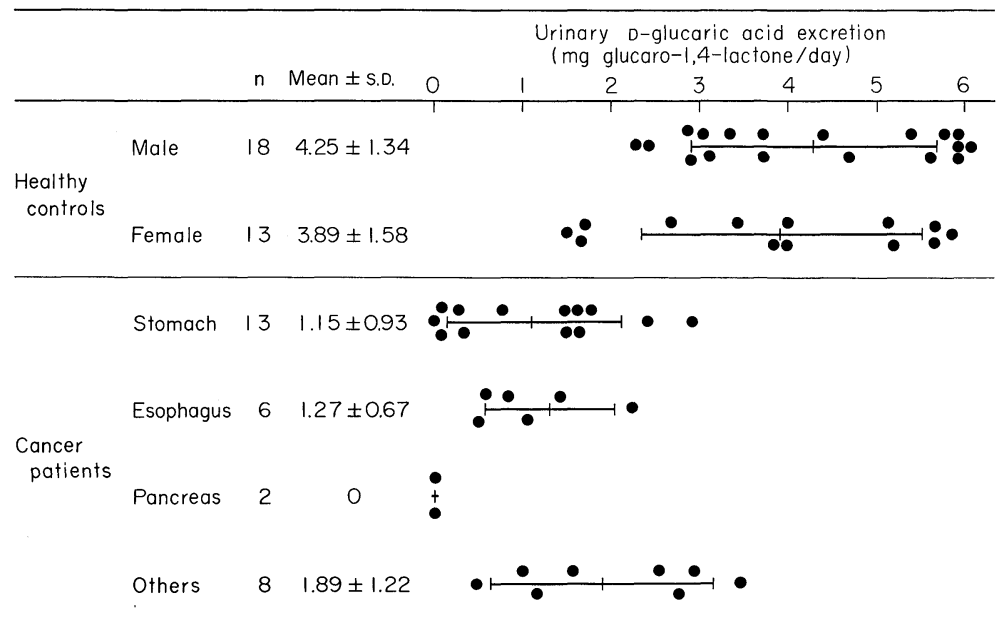

Fig. 2. Urinary D-glucaric acid excretion in healthy controls and in cancer patients.
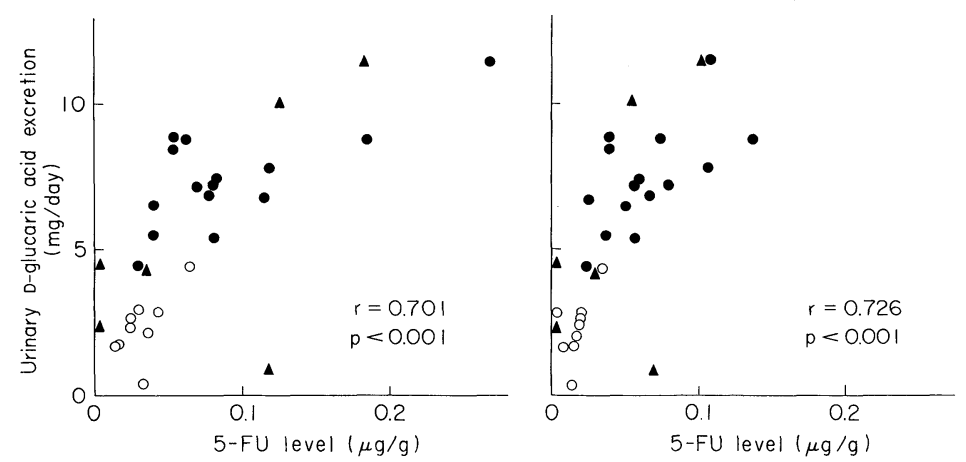

Fig. 3. Correlation between the level of D-glucaric acid in urine (mg glucaro-1,4-lactone/ day) and the degree of FT activation in cancer patients. The degree of FT activation is expressed as 5-FU levels in blood after i.v. administration of $800 \mathrm{mg}$ FT. Left: $1 \mathrm{hr}$

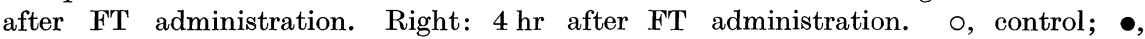
pretreated with phenobarbital; $\Delta$, pretreated with other drugs. 

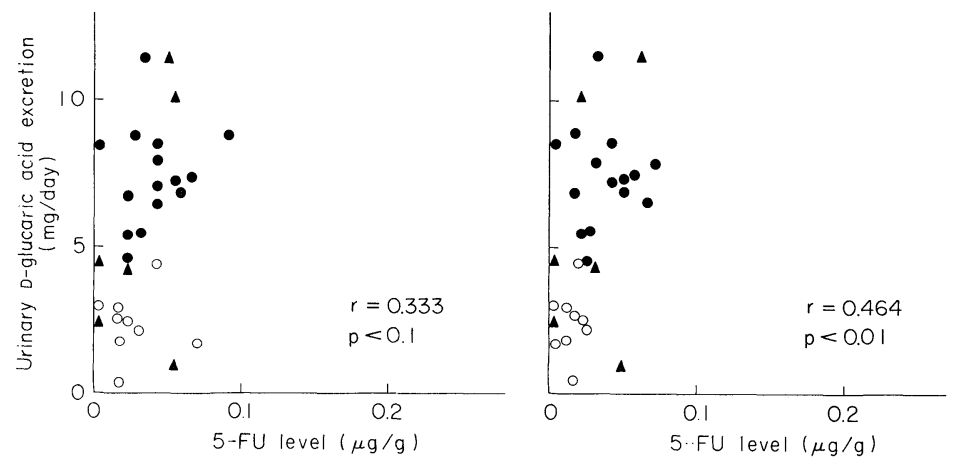

Fig. 4. Correlation between the level of $\mathrm{D}$-glucaric acid in urine and the degree of FT activation in cancer patients. See Fig. 3. Left: $8 \mathrm{hr}$ after FT administration. Right: $12 \mathrm{hr}$ after FT administration.

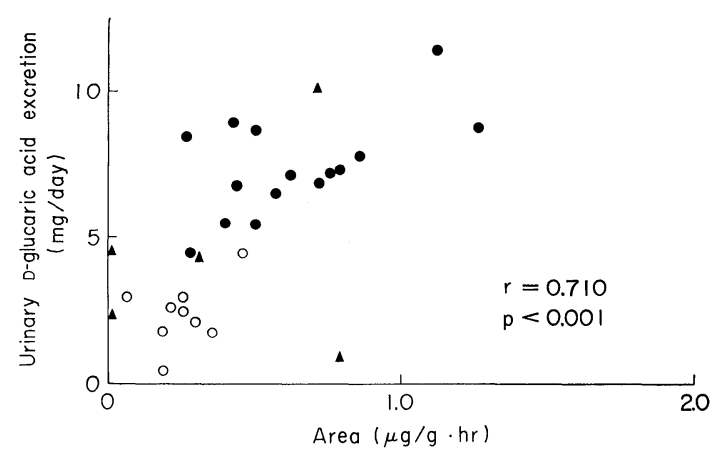

Fig. 5. Correlation between the level of D-glucaric acid in urine and the degree of FT activation in cancer patiens. FT activation is expressed as area under serum concentration of 5 -FU, 1,4,8, and $12 \mathrm{hr}$ after i.v. administration of $800 \mathrm{mg}$ FT. O, control; $\bullet$, pretreated with phenobarbital; $\Delta$, pretreated with other drugs.

of 5 -FU at $1,4,8$ and $12 \mathrm{hr}$ after administration of FT-207 was statistically significant ( $n=30, r=0.710, p<0.001$, Fig. 5).

\section{Cases studies}

Case 1. A 23-year-old female with stomach cancer (Fig. 6). The levels of urinary D-glucaric acid and serum FT-207 and 5-FU were measured according to the methods as described in Materials and Methods at 1, 4, 8 and $12 \mathrm{hr}$ after administration of FT-207 alone or in combination with phenobarbital. The urinary level of D-glucaric acid after pretreatment with phenobarbital increased about two-fold over that before pretreatment. The blood concentration of 5-FU after pretreatment increased about five- to six-fold over that before pretreatment during the period of estimation.

Case 2. A 73-year-old female with stomach cancer (Fig. 7). She had been on a combined regimen of phenobarbital and FT-207 for 8 months in our Hospital. 


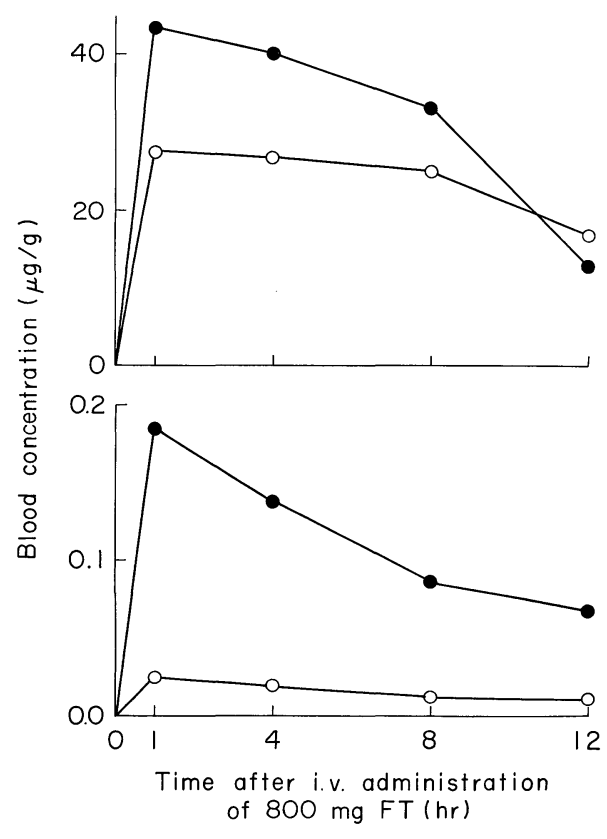

Fig. 6. Blood concentration of FT (upper panel) or its active metabolite, 5-FU, (lower panel) in a cancer patient pretreated with phenobarbital. (Patient H.N. 23 years old female with

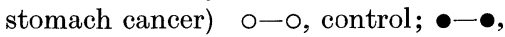
pretreated with phenobarbital (200 $\mathrm{mg} /$ day, for 3 days). The urinary excretion of D-glucaric acid was 2.62 $\mathrm{mg} /$ day in control and $9.65 \mathrm{mg} /$ day after treatment with phenobarbital.

H.S. $73 y$ female, stomach cancer

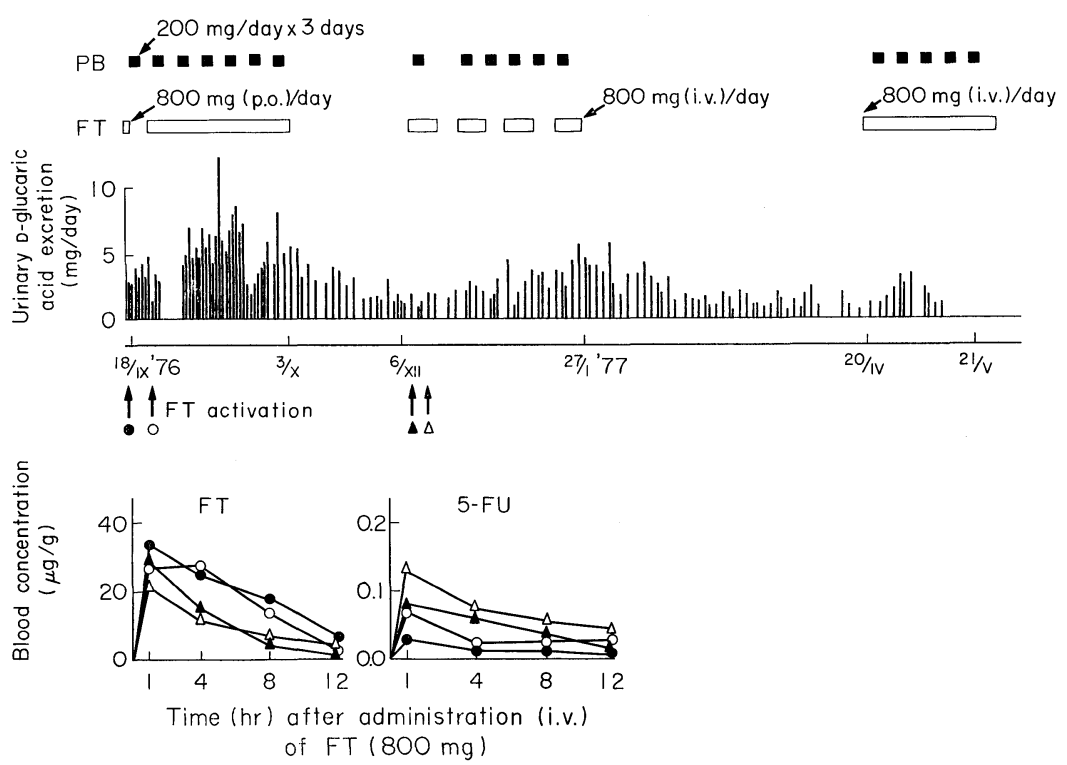

Fig. 7. Blood concentration of FT or its active metabolite, 5-FU, in a cancer patient pretreated with phenobarbital. 
Phenobarbital was given by an intramuscular injection in a daily dose of $200 \mathrm{mg}$ for three days weekly, and FT-207 by an intravenous infusion or orally in a daily dose of $800 \mathrm{mg}$ every day. The urinary level of D-glucaric acid increased after treatment with phenobarbital. It gradually decreased during the period without pretreatment. When she was in the final stage of stomach cancer, the urinary $\mathrm{D}^{-}$ glucaric acid level became lower. It then gradually increased after treatment with phenobarbital. When phenobarbital was discontinued, however, it started to decrease again.

\section{Discussion}

It has already been demonstrated that the FT-207-P-450 difference spectrum in the rat liver microsomes belongs to type II of Schenkman's classification, and that this difference spectrum of FT-207 had an absorption peak at $420 \mathrm{~nm}$ and a trough at $385 \mathrm{~nm}(\mathrm{Ks}=26.3 \mathrm{mM})$ (Ohira et al. 1976b).

This result shows that FT-207 is metabolized and activated in the NADPHrequiring monooxygenase reaction of cytochrome $\mathrm{P}-450$, a liver microsomal drugmetabolizing enzyme.

Also, a decrease of the activity of cytochrome P-450 has been observed in the tumor-bearing hosts.

It is anticipated that the application of the methods to elevate the activity of cytochrome P-450 is of advantage in the administration of masked compounds, such as FT-207 which does not show an anticancer activity before it is metabolized by cytochrome P-450.

Thus considered, it is highly recommendable to pretreat the cancer patients with phenobarbital which increases the activity of cytochrome P-450. In clinical practice the authors have been using the combined regimen of FT-207 and phenobarbital (Ohira et al. 1976a, 1978).

Therefore, when masked compounds are administered, it is important to follow the fluctuation of the daily activity of cytochrome P-450. As to the measurement of the activity of cytochome P-450, such methods, as punch biopsy, needle biopsy and autopsy have been reported (Kitagawa and Kamataki 1976).

Recently a sensitive method for the quantitative estimation was introduced (Sato et al. 1976), but in fact it will be impossible in cancer patients to carry out liver biopsy repeatedly. In the present communication it was investigated whether the measurement of urinary level of D-glucaric acid could be of value as an indicator for the evaluation of the activity of cytochrome P-450.

According to Aarts (1965), drugs such as phenobarbital which are known to cause induction of drug-metabolizing enzymes increase the urinary level of $\mathbf{D}$ glucaric acid. Presumably the increase of the urinary D-glucaric acid level is caused by an induction of UDPG dehydrogenase, because the activity of glucuronolactone dehydrogenase (Fig. 1) was not increased by the administration of phenobarbital. Therefore, it is conceivable that the urinary D-glucaric acid level reflects the activity of UDPG dehydrogenase, and that of UDP glucuronyl 
transferase (Hunter et al. 1973; Williams et al. 1973; Sotaniemi et al. 1974).

It seems likely that the drugs such as phenobarbital which are known to cause an induction of microsomal enzymes increase the urinary D-glucaric acid level as the result of the similar effects on the glucuronic acid pathway. In fact it has been reported that the activity of UDP glucuronyl transferase was enhanced by the administration of phenobarbital (Catz and Yaffe 1962).

In general, lipid-soluble drugs are made hydrophilic in the first phase of drug metabolism by oxidation, reduction, hydroxylation and by other processes. They are then conjugated to glucuronic acid, amino acid and so on which are the ordinary components of a living organism (the second phase). They are then excreted rapidly into the urine. These lipid-soluble drugs are detoxified or metabolized in the microsome, mitochondria and soluble fraction of the liver cells.

The microsomal enzymes play an important role in both the first and the second phases of drug metabolism. Namely, cytochrome P-450 is concerned with the first phase and UDP glucuronyl transferase is concerned with the second phase. The UDP glucuronyl transferase participates in the conjugation of glucuronic acid, which is one of the components in the glucuronic acid pathway as shown in Fig. 1 (Hunter et al. 1973).

D-Glucaric acid is regarded as the end-product of this pathway in men and guinea pigs. Accordingly, the urinary level of $\mathrm{D}$-glucaric acid is not only a direct indicator of the second phase, but also an indirect indicator of the first phase, namely an indicator of the activity of cytochrome P-450.

As described previously, Hunter et al. (1973) have already shown that there is a statistically significant correlation between the total liver content of cytochrome P-450 and the urinary level of D-glucaric acid. Therefore, the urinary level of D-glucaric acid may be a useful indicator to check the activity of cytochrome P-450 when a patient is being treated with a combined regimen of phenobarbital and masked compound such as FT-207.

It was shown previously that the activity of drug-metabolizing enzymes, e.g. cytochrome P-450, decreased gradually with time in various tumor-bearing (YS, $\mathrm{AH}$ 109A, and AH130) rats (Ohira 1977). For instance, the level of cytochrome P-450 in YS-bearing rats decreased gradually as evidenced by determinations at $3,5.5$ and 6.5 days after inoculation and it was about $1 / 10$ of that in normal controls in the last stage. The drug-metabolizing activity, such as aminopyrine $\mathrm{N}$ demethylation and aniline hydroxylation, decreased. Almost in parallel with the gradual decrease of cytochrome $\mathrm{C}$ reductase and cytochrome $b_{5}$, the rate of activation of FT-207 and cyclophosphamide also decreased. In other tumor-bear-

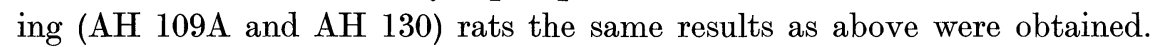

On the other hand, the urinary $\mathrm{D}$-glucaric acid level in cancer patients was distinctly lower than that in normal controls as shown in Fig. 2. And also, in cancer patients the blood level of 5 -FU which is an active metabolite of FT-207 was measured with intervals of $1 \mathrm{hr}$ after administration of FT-207. As a result, the level of 5-FU under pretreatment of phenobarbital was found to be higher than 
that in those who received no pretreatment of phenobarbital (Ohira 1977).

The correlation between the urinary D-glucaric acid level and the degree of FT-207 activation was statistically significant as shown in Figs. 3, 4 and 5. It was thus concluded in the present investigation that the urinary level of D-glucaric acid was an indirect indicator for the evaluation of the degree of the activity of cytochrome P-450.

It has been demonstrated that the correlation between the urinary level of $\mathrm{D}$ glucaric acid and the rate of plasma clearance of pentobarbital in various liver diseases is statistically significant and that the urinary level of D-glucaric acid increases after administration of pentobarbital (Yamamoto 1976).

The drug-metabolizing activity in liver diseases is maintained at a normal level during the acute phase. But it has been suggested that the rate of plasma clearance of antipyrine is reduced during the acute phase of hapatitis, while the urinary $\mathrm{D}$-glucaric acid excretion is paradoxically high. Thus, the use of urinary D-glucaric acid level as an index of microsomal enzyme induction has been shown to be unreliable in the presence of liver injury (Sorrel et al. 1976).

In this report cancer patients selected for study had no liver metastases. In fact, however, the glucuronic acid pathway is very complex and some of the enzymes involved are not detected in the microsome but in the cytosol. Furthermore, the rate-limiting step of this pathway is unknown. As described above, the urinary level of D-glucaric acid may be an indicator of the rate of induction of drug-metabolizing enzymes. However, the significance of the urinary level of D-glucaric acid is not yet fully elucidated at present. It will be necessary to investigate in detail the correlation between the urinary level of D-glucaric acid and various backgrounds of the patients; for example, stage, extent of liver metastases and so forth.

On the other hand, it will be necessary to investigate in rats the correlation between the level of cytochrome P-450 and urinary level of L-ascorbic acid and D-glucaric acid, because rats are able to synthesize both L-ascorbic acid and Dglucaric acid (Aarts 1965).

\section{References}

1) Aarts, E.M. (1965) Evidence for the function of D-glucaric acid as an indicator for drug induced enhanced metabolism through glucuronic acid pathway in man. Biochem. Pharmacol., 14, 359-363.

2) Catz, C. \& Yaffe, S.J. (1962) Pharmacological modification of bilirubin conjugation in the newborn. Amer. J. Dis. Child., 104, 516-517.

3) Fujita, H., Ogawa, K., Sawabe, T. \& Kimura, K. (1972) Metabolism of N1-(2'tetrahydrofuryl)-5-fluorouracil. Jap. J. Cancer Clin., 18, 917-922.

4) Hunter, J., Maxwell, J.D., Carrela, M., Stewart, D.A. \& Williams, R. (1973) Urinary D-glucaric acid excretion and total liver content of cytochrome P-450 in guinea pigs: Relationship during enzyme induction and following inhibition of protein synthesis. Biochem. Pharmacol., 22, 743-747.

5) Kitagawa, H. \& Kamataki, T. (1976) Drug metabolism and cytochrome P-450. Metabolism and Disease, 13, 1691-1703. (Japanese)

6) Marsh, C.A. (1963) Metabolism of glucuronolactone in mammalian systems. Identification of D-glucaric acid as a normal constitutent of urine. Biochem. J., 86, 77-86. 
7) Ohira, S. (1977) Cancer chemotherapy in relation to the level of microsomal drugmetabolizing enzymes (cytochrome P-450) in the liver. Jap. J. Cancer Clin., 23, 720733.

8) Ohira, S., Maezawa, S., Watanabe, K., Kitada, K. \& Saito, T. (1976a) Experimental approach to increase the effects of cancer chemotherapy in tumor-bearing rats pretreated with an inducer on microsomal drug-metabolizing enzyme (cytochrome P-450). Chemotherapy (N.Y.), 8, 197-202.

9) Ohira, S., Maezawa, S., Irinoda, Y., Watanabe, K., Kitada, K., Sakuma, M. \& Saito, T. (1976b) Studies on the cytochrome P-450 difference spectra induced by various anti-cancer drugs by liver microsomes from rats. Cancer and Chemotherapy., 3, 663669.

10) Ohira, S., Maezawa, S., Watanabe, K., Kitada, K., Sakuma, M., Matsuoka, S. \& Saito, T. (1978) Cancer chemotherapy with FT-207 in combination with an inducer of microsomal cytochrome P-450 and an inhibitor of microsomal lipid peroxidation. In: Current Chemotherapy: Proceeding of the 10th International Congress of Chemotherapy, edited by W. Siegenthaler \& R. Lüthy, American Society for Microbiology, Washington, D.C., pp. 1161-1164.

11) Sato, N., Hagihara, B., Kameda, T. \& Abe, H. (1976) A sensitive method for the quantitative estimation of cytochromes $\mathrm{a}$ and $\mathrm{a}_{3}$ in tissues. Analyt. Biochem., 74, 105-117.

12) Sorrel, M.F., Burnett, D.A., Tuma, D.J. \& Barak, A.J. (1976) Paradoxical urinary excretion of D-glucaric acid in acute viral hepatitis. Clin. Pharmacol. Ther., 20, $365-368$.

13) Sotaniemi, E.A., Medzihradsky, F. \& Eliasson, G. (1974) Glucaric acid as an indicator of use of enzyme-inducing drugs. Clin. Pharmacol. Ther., 15, 417-423.

14) Williams, R., Hunter, J. \& Maxwell, J.D. (1973) Measurement of hepatic enzyme induction in man. Use of D-glucaric acid excretion test. In: The Liver: Quantitative Aspects of Structure and Function, edited by G. Paumgartner \& R. Preisig, Karger, Berne, pp. 224-231.

15) Yamamoto, S. (1976) Clinical Aspects for Drug-Induced Liver Disease. Kanehara Shuppan, Tokyo, pp. 42-43. (Japanese) 